\title{
Study of Electron Beam on Electron Cyclotron Waves with AC Field in the Magnetosphere of Uranus
}

\author{
Rajbir Kaur and R.S. Pandey \\ Department of Physics, Amity Institute of Applied Sciences, Amity University \\ Sector -125, Noida, Uttar Pradesh, India \\ *corresponding author, E-mail: rspandey@amity.edu
}

\begin{abstract}
In this paper, we investigate the electromagnetic electron cyclotron (EMEC) waves in the magnetosphere of Uranus. By using the method of characteristic solution, the expression for dispersion relation is drawn. Following kinetic approach, the growth rate and real frequency of EMEC waves is studied theoretically, considering the injection of cold plasma beam in the Uranian system. The observations made by a space probe launched by NASA, Voyager 2, showed unusual orientation of planet's spin axis and presence of more particles in high energy tail in Uranian magnetospheric plasma. Therefore, in this paper Kappa distribution is employed instead of usual Maxwellian distribution. The study is extended to the parallel as well as the oblique propagation of EMEC waves with variation in temperature anisotropy, number density of electrons and angle of propagation with respect to magnetic field direction. It is found that these parameters support the growth rate of EMEC waves. But response of real frequency of these waves is not same as that of growth rate for all the cases. Numerical analysis also revealed that as the ratio of number density of cold to hot plasma increases growth rate of EMEC waves also increases. Thus, denser the beam is injected, more the growth can be observed. These results are appropriate for applications to space plasma environments and magnetospheric regimes for detailed comparative planetary study.
\end{abstract}

Keywords: Space Plasma, Uranus, Magnetosphere, Electroncyclotron Waves

\section{Introduction}

The Voyager 2 flyby of Uranus revealed that the planet has a large and unusual magnetosphere. The magnetic field experiment carried on Voyager 2 [1] reported the maximum magnitude of magnetic field as 413 nanotesla, observed at 4.19 $\mathrm{R}_{\mathrm{U}}$, just before the closest approach to Uranus [2]. The unique feature of Voyager 2 encounter was the fact that the angle between Uranus's angular momentum vector and dipole moment vector has the large value of 60 degrees. So the spin axis of Uranus is aligned nearly along the planet-sun axis. This leads to the situation that the flow system rotational electric field is oriented in such a way that instead of shielding the middle magnetosphere from the solar wind, it allows solar wind effects to deeply penetrate into the magnetosphere of Uranus [3]. As the consequences of this, Uranian system permits us to study magnetospheric instabilities and strong dynamics including Earth like injection phenomena. Therefore, in present paper we have conducted the theoretical investigation to find the behavior of various parameters when cold plasma beam is injected in the magnetosphere of Uranus.

The Plasma Wave Instrument carried by Voyager 2 detected significant phenomenas related to local wave-particle interactions, radio emissions, electrostatic Bernstein waves and plasma waves [4-10]. Gurnett and his team also reported the presence of electron cyclotron waves in the region of inner magnetosphere of Uranus [3]. They are believed to be generated by cyclotron resonance interaction with energetic electrons. From the anisotropy produced by the loss cone distribution of trapped electrons, comes the free source of energy. The electromagnetic electron cyclotron instability can be considered as one of the most important topic to study in space physics. Literature presents electron cyclotron harmonics $(\mathrm{ECH})$ waveparticle interaction as the major source of energy diffusion and pitch-angle diffusion in magnetospheres of planets. These diffusions, because of growth of waves, leading to electron precipitation are enough to generate diffuse auroral emissions detected by spacecrafts in planetary magnetospheres [5, 11-14].

The Cosmic Ray System on Voyager 2 measured the significant fluxes of energetic protons and electrons in those regions of magnetospheres of planets where these particles were expected to be stably trapped $[15,16]$. [17] considered velocity space diffusion for waves propagating at an arbitrary angle to magnetic field. In a similar way, theory of resonant interaction between energetic charged particle and whistler cyclotron waves propagating oblique to non-uniform geomagnetic field in inhomogenous plasma was studied [18]. The work considered Hamiltonian approach to analyze particle equation of motion. In contrast to this methodology, we have adapted kinetic 
approach to investigate particle equation of motion, in present study. A detailed review on the kinetic electromagnetic instabilities and importance of this approach, provided by [19], is referred. It dealt with effect of cold plasma addition, variation of growth rates, acceleration of heavier ions and results of computer simulation experiments explaining the evolution of particle distribution function and wave activity. The studies show that ion cyclotron noise couples to ions and similarly whistler mode noise leads to electron pitch angle diffusion. This diffusion results in particle precipitation and creates a distribution of trapped particles that is unstable to further wave growth [17].

Low-Energy Charged-Particle (LECP) instrument on Voyager 2 revealed that magnetospheric particle population principally consists of electrons and protons having energies to at least 1.2 to $4 \mathrm{MeV}$, respectively. And electron intensities substantially exceed proton intensities at a given energy [20]. The evidences given by Plasma Science Experiment on Voyager 2 suggested that the proton distribution functions at Uranus must be characterized by a "warm" (i.e., subsonic) core and a nonMaxwellian tail that varied significantly along the spacecraft trajectory. The non-Maxwellian tail carried most of the plasma energy density in the observed energy range $(<6 \mathrm{keV})$, which is small compared to the energy density of the planetary magnetic field. [21]. Therefore, on the basis of above mentioned observation, we concluded that natural space plasmas in the magnetosphere of Uranus possess a pronounced nonMaxwellian tail distribution that can be well studied by kappa distributions. So, kappa distribution is employed instead of usual Maxwellian distribution for particles [22, 23]. Kappa distribution functions have high energy tails decreasing as a power law in particle speed, deviating from Maxwellian distribution. The results of work done by [24] illustrates how kappa function degenerates to Maxwellian. They have used spectral index ' $k$ ' that determines slope of energy spectrum of superathermal particles and its variation shows that in the limit $\mathrm{k} \rightarrow \infty$, kappa distribution behaves like Maxwellian distribution.

Also [25] investigated the process of generation of electric field and its magnitude in the range of $1-10 \mathrm{mV} / \mathrm{m}$ in the atmosphere of Uranus. Their study concluded that the dynamo-driven magnetic field aligned current in the ionosphere produce plasma instabilities which produces increase in resistivity with a resulting electric field. In presence of similar perpendicular AC electric field of Uranus, [26] investigated whistler mode waves for subtracted bi-Maxwellian plasma in the magnetosphere [27]. Analyzing the growth rate, showed that either the loss-cone or the temperature anisotropy in the hot plasma component leads to generation of low frequency whistler mode emissions. Also, the case of temporal evolution of whistler instability due to cold plasma injection was studied by [28]. In their work, a generalized distribution function with index $\mathrm{j}$ was used for background warm plasma and time dependent injected cold plasma was considered with a simple Maxwellian distribution. Small amplitude oblique and parallel whistler mode waves were studied numerically by [29] with the help of electromagnetic Vlasov dispersion equation. Electromagnetic Vlasov results were compared with those of cold-plasma and electrostatic Vlasov approximations. It was concluded that near resonance, whistler mode has group velocity more likely parallel to magnetic field than that expected by cold-plasma theory. Literature reports that electron beam instability, the conventional ion acoustic instability, and the ion acoustic beam instability were studied considering the linear theory of electrostatic Vlasov instabilities. The work can be compared with the present work as it gave the detailed parametric analysis of beam instabilities [30].

In recent past, many researchers have explored various plasma regimes for instabilities using different distribution functions with different techniques. [31] applied temperature-dependent fluid model to study the oblique electromagnetic instabilities, driven by hot electron beam interacting with hot and magnetized plasma. [32] reported the generation of whistler emissions by injection of hot electron beam. [33] modelled anisotropic plasma with product-bi-kappa distribution to investigate instability of parallel electron whistler-cyclotron modes. [34, 35] discussed the results of growth rate for relativistic whistler mode waves using generalized distribution function with perpendicular AC field and kappa-loss-cone distribution function respectively. [36] studied the effect of kappa index and other parameters on obliquely propagating electromagnetic waves in kappa-Maxwellian plasma. [37], using methods of characteristic solutions, studied effect of beam for space plasma at magnetospheric height of 1A.U. [38] examined the effect of loss-cone distribution index $\mathrm{J}$ with temperature anisotropy for plasma-pause region.

Motivated from above mentioned developments, in this paper, we offer both parametric variations derived from numerical solutions of the electron-cyclotron instability as well as analytical treatment of the problem which succeeds in unifying the various numerical results. By using the method of characteristic solution and kappa distribution function, the expression for dispersion relation is drawn. Following kinetic approach, the growth rate of EMEC waves and their real frequency is studied theoretically, considering the injection of cold plasma beam in the Uranian system. The study is extended to the parallel as well as the oblique propagation of EMEC waves with variation in temperature anisotropy, energy density of electrons and angle of propagation with respect to magnetic field direction.

\section{Mathematical Formulation}

A spatially homogeneous anisotropic, collisionless plasma subjected to external magnetic field $\mathbf{B}_{\mathbf{o}}=\mathbf{B}_{\mathrm{o}} \hat{\mathbf{e}}_{\mathbf{z}}$ and an electric field $\mathbf{E}_{\mathbf{o}}=\mathrm{E}_{\mathrm{o}} \sin (\mathrm{v} \mathrm{t}) \hat{\mathbf{e}}_{\mathbf{x}}$ has been considered to get dispersion relation. In this case, linearized Vlasov-Maxwell equations, obtained after neglecting higher order terms and 
separating the equilibrium and non-equilibrium parts, following the technique of $[32,39]$, are given as below:

$$
\begin{aligned}
& \mathbf{v} \cdot\left(\frac{\partial \mathrm{f}_{\mathrm{so}}}{\partial \mathbf{r}}\right)+\frac{\mathrm{e}_{\mathrm{s}}}{\mathrm{m}_{\mathrm{s}}}\left[\mathrm{E}_{\mathrm{o}} \sin (\mathrm{v} \mathrm{t})+\left(\mathbf{v} \times \mathbf{B}_{\mathrm{o}}\right)\right]\left(\frac{\partial \mathrm{f}_{\mathrm{so}}}{\partial \mathbf{v}}\right)=0 \\
& \frac{\partial \mathrm{f}_{\mathrm{s} 1}}{\partial \mathrm{t}}+\mathbf{v} \cdot\left(\frac{\partial \mathrm{f}_{\mathrm{s} 1}}{\partial \mathbf{r}}\right)+\left(\mathrm{F} / \mathrm{m}_{\mathrm{s}}\right) \frac{\partial \mathrm{f}_{\mathrm{s} 1}}{\partial \mathbf{v}}=\mathrm{S}(\mathbf{r}, \mathbf{v}, \mathrm{t})
\end{aligned}
$$

Where force is given as $\mathrm{F}=\mathrm{m} \frac{\mathrm{dv}}{\mathrm{dt}}$

$$
\mathbf{F}=\mathrm{e}_{\mathrm{s}}\left[\mathrm{E}_{\mathrm{o}} \sin (v \mathrm{t})+\left(\mathbf{v} \times \mathbf{B}_{\mathrm{o}}\right)\right]
$$

The particle trajectories are obtained by solving equation of motion defined in equation (3) and $\mathrm{S}(\mathrm{r}, \mathrm{v}, \mathrm{t})$ is defined as:

$$
\mathrm{S}(\mathbf{r}, \mathbf{v}, \mathrm{t})=\left(\mathrm{e} / \mathrm{m}_{\mathrm{s}}\right)\left[\mathrm{E}_{1}+\left(\mathbf{v} \times \mathbf{B}_{\mathbf{1}}\right)\right]\left(\frac{\partial \mathrm{f}_{\mathrm{so}}}{\partial \mathbf{v}}\right)
$$

where $\mathrm{s}$ denotes species and $\mathrm{E}_{1}, \mathrm{~B}_{1}$ and $\mathrm{f}_{\mathrm{s} 1}$ are perturbed quantities and are assumed to have harmonic dependence in $\mathrm{E}_{1}$, $\mathrm{B}_{1}$ and $\mathrm{f}_{\mathrm{s} 1}=\exp \mathrm{i}(\mathbf{k} \cdot \mathbf{r}-\omega \mathrm{t})$.

The method of characteristic solution is used to determine the perturbed distribution function, fs 1 , which is obtained from Eq. (2) by

$\mathrm{f}_{\mathrm{s} 1}(\mathbf{r}, \mathbf{v}, \mathrm{t})=\int_{0}^{\infty} \mathrm{s}\left\{\mathbf{r}_{\mathbf{o}}\left(\mathbf{r}, \mathbf{v}, \mathrm{t}^{\prime}\right), \mathbf{v}_{\mathbf{o}}\left(\mathbf{r}, \mathbf{v}, \mathrm{t}^{\prime}\right), \mathrm{t}-\mathrm{t}^{\prime}\right\} \mathrm{dt}^{\prime}$

The phase space coordinate system has been transformed from $(\mathbf{r}, \mathbf{v}, \mathrm{t})$ to $\left(\mathbf{r}_{\mathbf{o}}, \mathbf{v}_{\mathbf{o}}, \mathrm{t}-\mathrm{t}^{\prime}\right)$. The particle trajectories which are obtained by solving eq. (3) for the given external field and wave propagation, $\mathbf{k}=\left[\mathbf{k}_{\perp} \hat{\mathrm{e}}_{\mathrm{x}}, 0, \mathbf{k}_{\|} \hat{\mathrm{e}}_{\mathrm{z}}\right]$ are:

$\mathbf{x}_{\mathbf{0}}=\mathbf{x}+\left(\frac{\mathbf{v}_{\mathbf{y}}}{\omega_{c s}}\right)+\left(\frac{1}{\omega_{c s}}\right)\left[\mathbf{v}_{\mathbf{x}} \sin \omega_{c s} t^{\prime}-\mathbf{v}_{\mathbf{y}} \cos \omega_{c s} t^{\prime}\right]+\left(\frac{\Gamma_{x}}{\omega_{c s}}\right)\left[\frac{\omega_{c s} \sin v t^{\prime}-v \sin \omega_{c s} t^{\prime}}{\omega_{c s}^{2}-v^{2}}\right]$

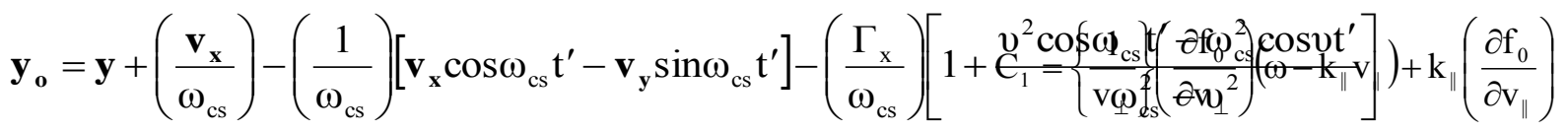

$$
\mathbf{z}_{\mathbf{o}}=\mathbf{z}-\mathbf{v}_{\mathbf{z}} \mathbf{t}^{\prime}
$$

And the velocities are

$$
\mathbf{v}_{\mathbf{x o}}=\mathbf{v}_{\mathbf{x}} \cos \omega_{\mathrm{cs}} \mathbf{t}^{\prime}-\mathbf{v}_{\mathbf{y}} \sin \omega_{\mathrm{cs}} \mathrm{t}^{\prime}+\left\{\frac{v \Gamma_{\mathrm{x}}\left(\cos v \mathrm{t}^{\prime}-\cos \omega_{\mathrm{cs}} \mathrm{t}^{\prime}\right)}{\omega_{\mathrm{cs}}^{2}-v^{2}}\right\}
$$

$$
\mathbf{v}_{\mathbf{y} \mathbf{0}}=\mathbf{v}_{\mathbf{x}} \sin \omega_{\mathrm{cs}} \mathrm{t}^{\prime}+\mathbf{v}_{\mathbf{y}} \cos \omega_{\mathrm{cs}} \mathrm{t}^{\prime}-\left\{\frac{\Gamma_{\mathrm{x}}}{\left(\omega_{\mathrm{cs}} \sin v \mathrm{t}^{\prime}-v \sin \omega_{\mathrm{cs}} \mathrm{t}^{\prime}\right)} \omega_{\mathrm{cs}}^{2}-v^{2}\right\}
$$

$\mathbf{v}_{\mathrm{zo}}=\mathbf{v}_{\mathrm{z}}$

Where $\omega_{c s}=\frac{e_{s} B_{o}}{m_{s}}=$ cyclotron frequency of species $\mathrm{s}$

$$
\begin{aligned}
& \Gamma_{\mathrm{x}}=\frac{\mathrm{e}_{\mathrm{s}} \mathrm{E}_{\mathrm{o}}}{\mathrm{m}_{\mathrm{s}}}=\mathrm{AC} \text { electric field varying as } \\
& \mathrm{E}=\mathrm{E}_{\mathrm{ox}} \sin (v \mathrm{t}) \\
& \mathrm{v}=\text { angular AC frequency }
\end{aligned}
$$

After some algebraic simplifications and integration, the perturbed distribution function is given as:

$$
\begin{aligned}
\mathrm{f}_{\mathrm{s} 1}(\mathbf{r}, \mathbf{v}, \mathrm{t})= & -\frac{\mathrm{e}_{\mathrm{s}}}{\mathrm{m}_{\mathrm{s}} \omega} \sum_{\mathrm{m}, \mathrm{n}, \mathrm{p}, \mathrm{q}}^{\infty} \frac{\mathrm{J}_{\mathrm{p}}\left(\lambda_{2}\right) \mathrm{J}_{\mathrm{m}}\left(\lambda_{1}\right) \mathrm{J}_{\mathrm{q}}\left(\lambda_{3}\right) \mathrm{e}^{\mathrm{i}(\mathrm{k} . \mathrm{r}-\omega \mathrm{t})}}{\left.\mathbf{v}_{\|}-(\mathrm{n}+\mathrm{q}) \omega_{\mathrm{cs}}+\mathrm{pv}\right\}^{\times}} \times \\
& {\left[\mathrm{E}_{1 \mathrm{x}} \mathrm{J}_{\mathrm{n}} \mathrm{J}_{\mathrm{p}}\left\{\left(\frac{\mathrm{n}}{\lambda_{1}}\right) \mathrm{U}^{*}+\mathrm{D}_{1}\left(\frac{\mathrm{p}}{\lambda_{2}}\right)\right\}-\right.} \\
& \left.\mathrm{iE}_{1 \mathrm{y}}\left\{\mathrm{J}_{\mathrm{n}}^{\prime} \mathrm{J}_{\mathrm{p}} \mathrm{C}_{1}+\mathrm{J}_{\mathrm{n}} \mathrm{J}_{\mathrm{p}}^{\prime} \mathrm{D}_{2}\right\}+\mathrm{E}_{1 \mathrm{x}} \mathrm{J}_{\mathrm{n}} \mathrm{J}_{\mathrm{p}} \mathrm{W}^{*}\right]
\end{aligned}
$$

Where $\mathrm{e}^{\mathrm{i} \lambda(\sin \theta)}=\sum_{\mathrm{k}=-\infty}^{\infty} \mathrm{J}_{\mathrm{k}}(\lambda) \mathrm{e}^{\mathrm{i} k \theta}$ is the Bessel identity. It has been used as an argument of the following functions:

$$
\lambda_{1}=\frac{\mathbf{k}_{\perp} \mathbf{v}_{\perp}}{\omega_{\mathrm{cs}}}, \lambda_{2}=\frac{\mathbf{k}_{\perp} \Gamma_{\mathrm{x}}}{\omega_{\mathrm{cs}}^{2}-v^{2}}, \lambda_{3}=\frac{\mathbf{k}_{\perp} \Gamma_{\mathrm{x}} v}{\omega_{\mathrm{cs}}^{2}-v^{2}}
$$




$$
\begin{aligned}
& \mathrm{W}^{*}=\left[\left(\mathrm{n} \omega_{\mathrm{cs}} \frac{\mathbf{v}_{\|}}{\mathbf{v}_{\perp}}\right)\left(\frac{\delta \mathrm{f}_{0}}{\delta \mathbf{v}_{\perp}}\right)-\mathrm{n} \omega_{\mathrm{cs}}\left(\frac{\delta \mathrm{f}_{\mathrm{o}}}{\delta \mathbf{v}_{\perp}}\right)\right]+\left\{1+\left\{\frac{\mathbf{k}_{\perp} \Gamma_{\mathrm{x}} v}{\omega_{\mathrm{cs}}^{2}-v^{2}}\right\}\left\{\frac{\mathrm{p}}{\lambda_{2}}-\frac{\mathrm{n}}{\lambda_{1}}\right\}\right] \\
& \mathrm{D}_{1}=\mathrm{C}_{1}\left(\frac{\mathrm{v} \Gamma_{\mathrm{x}}}{\omega_{\mathrm{cs}}^{2}-v^{2}}\right), \quad \mathrm{D}_{2}=\mathrm{C}_{1}\left(\frac{\omega_{\mathrm{cs}} \Gamma_{\mathrm{x}}}{\omega_{\mathrm{cs}}^{2}-v^{2}}\right) \\
& \mathrm{J}_{\mathrm{n}}^{\prime}=\frac{\mathrm{d} \mathrm{J}_{\mathrm{n}}\left(\lambda_{1}\right)}{\mathrm{d} \lambda_{1}}, \quad \mathrm{~J}_{\mathrm{p}}^{\prime}=\frac{\mathrm{d} \mathrm{J}_{\mathrm{p}}\left(\lambda_{2}\right)}{\mathrm{d} \lambda_{2}}
\end{aligned}
$$

The conductivity tensor is written as:

$$
\|\boldsymbol{\sigma}\|=-\Sigma\left(\frac{\mathrm{e}_{\mathrm{s}}}{\mathrm{m}_{\mathrm{s}} \omega}\right) \sum_{\mathrm{m}, \mathrm{n}, \mathrm{p}, \mathrm{q}=-\infty}^{\infty} \int \mathrm{d}^{3} \mathbf{v}\left[\frac{\mathrm{J}_{\mathrm{q}}\left(\lambda_{3}\right) \mathrm{S}_{\mathrm{ij}}}{\omega-\mathbf{k}_{\|} \mathbf{v}_{\|}-(\mathrm{n}+\mathrm{q}) \omega_{\mathrm{cs}}+\mathrm{pv}}\right]
$$

$$
\|\mathbf{S}\|=\left|\begin{array}{ccc}
\mathbf{v}_{\perp} \mathrm{J}_{\mathrm{n}}^{2} \mathrm{~J}_{\mathrm{p}}\left(\frac{\mathrm{n}}{\lambda_{1}}\right) \mathrm{A} & \mathrm{i} \mathbf{v}_{\perp} \mathrm{J}_{\mathrm{n}} \mathrm{B} & \mathbf{v}_{\perp} \mathrm{J}_{\mathrm{n}}^{2} \mathrm{~J}_{\mathrm{p}}\left(\frac{\mathrm{n}}{\lambda_{1}}\right) \mathrm{W} * \\
\mathbf{v}_{\perp} \mathrm{J}_{\mathrm{n}}^{\prime} \mathrm{J}_{\mathrm{n}} \mathrm{J}_{\mathrm{p}} A & \mathbf{v}_{\perp} \mathrm{J}_{\mathrm{n}}^{\prime} \mathrm{B} & \mathrm{i} \mathbf{v}_{\perp} \mathrm{J}_{\mathrm{n}}^{\prime} \mathrm{J}_{\mathrm{n}} \mathrm{J}_{\mathrm{p}} \mathrm{W}^{*} \\
\mathbf{v}_{\|} \mathrm{J}_{\mathrm{n}}^{2} \mathrm{~J}_{\mathrm{p}} \mathrm{A} & \mathbf{v}_{\|} \mathrm{J}_{\mathrm{n}} \mathrm{B} & \mathbf{v}_{\|} \mathrm{J}_{\mathrm{n}}^{2} \mathrm{~J}_{\mathrm{p}} \mathrm{W}^{*}
\end{array}\right|
$$

$$
\begin{aligned}
\mathrm{A}=\left(\frac{\mathrm{n}}{\lambda_{1}}\right) \mathrm{U}^{*}+ & \left(\frac{\mathrm{p}}{\lambda_{1}}\right) \mathrm{D}_{1} \\
\mathrm{~B} & =\mathrm{J}_{\mathrm{n}}^{\prime} \mathrm{J}_{\mathrm{p}} \mathrm{C}_{1}+\mathrm{J}_{\mathrm{n}}^{\prime} \mathrm{J}_{\mathrm{n}} \mathrm{D}_{2}
\end{aligned}
$$

From $\boldsymbol{J}=\|\boldsymbol{\sigma}\| \boldsymbol{E}_{\mathbf{1}}$ and two Maxwell's curl equations for the perturbed quantities, the wave equations can be written as:

$\left[\mathbf{k}^{2}-\mathbf{k} \cdot \mathbf{k}-\left(\omega^{2} / \mathrm{c}^{2}\right) \in(\mathbf{k}, \omega)\right] \mathrm{E}_{1}=0$

Where $\|\in(\mathbf{k}, \omega)\|=1-(4 \pi / \mathbf{i} \omega)\|\boldsymbol{\sigma}(\mathbf{k}, \omega)\|=$ dielectric tensor

$\epsilon_{\mathrm{ij}}(\mathrm{k}, \omega)=1+\sum_{\mathrm{s}} \frac{4 \pi e_{\mathrm{s}}^{2}}{\mathrm{~m}_{\mathrm{s}} \omega^{2}} \sum_{\mathrm{n}} \sum_{\mathrm{p}} \mathrm{J}_{\mathrm{p}}\left(\lambda_{2}\right) \mathrm{J}_{\mathrm{q}}\left(\lambda_{3}\right) \int \frac{\mathrm{d}^{3} \mathrm{vS}_{\mathrm{ij}}}{\omega-\mathrm{k}_{\|} \mathrm{v}_{\|}-(\mathrm{n}+\mathrm{q}) \omega_{\mathrm{cs}}+\mathrm{pv}}$

The generalized dielectric tensor may be written as:

$$
\left|\begin{array}{ccc}
\mathrm{N}^{2} \cos ^{2} \theta_{1}+\varepsilon_{11} & \varepsilon_{12} & \mathrm{~N}^{2} \cos \theta_{1} \sin \theta_{1}+\varepsilon_{13} \\
\varepsilon_{21} & \mathrm{~N}^{2}+\varepsilon_{22} & \varepsilon_{23} \\
\mathrm{~N}^{2} \cos \theta_{1} \sin \theta_{1}+\varepsilon_{31} & \varepsilon_{32} & \mathrm{~N}^{2} \sin ^{2} \theta_{1}+\varepsilon_{33}
\end{array}\right|
$$

After using the limits in above tensor $\mathbf{k}_{\perp}=\mathrm{k} \sin \theta_{1} \rightarrow 0$ and $\mathbf{k}_{\|}=\mathrm{k} \cos \theta_{1}$, the generalized dielectric tensor becomes simplified tensor:

$$
\left|\begin{array}{ccc}
-\mathrm{N}^{2}+\varepsilon_{11} & \varepsilon_{12} & 0 \\
-\varepsilon_{21} & -\mathrm{N}^{2}+\varepsilon_{22} & 0 \\
0 & 0 & \varepsilon_{33}
\end{array}\right|
$$

For EMEC waves, it is rewritten in more convenient form:

$$
-\mathrm{N}^{4}-2 \varepsilon_{11} \mathrm{~N}^{2}+\varepsilon_{11}^{2}+\varepsilon_{12}^{2}=0
$$

Neglecting the higher order terms of $\mathrm{n}$, the relation becomes:

$$
\varepsilon_{11} \pm \varepsilon_{12}=\mathrm{N}^{2}
$$

The unperturbed Lorentzian-Kappa distribution function is:

$$
\mathrm{F}_{\mathrm{ok}}=\frac{\mathrm{n}_{\mathrm{o}}}{\pi^{3 / 2} \theta_{1}^{2} \boldsymbol{\theta}_{\|} \mathrm{k}^{3 / 2}} \frac{\Gamma(\mathrm{k}+1)}{\Gamma(\mathrm{k}+1 / 2)}\left[1+\frac{\mathbf{v}_{\|}^{2}}{\mathrm{k} \boldsymbol{\theta}_{\|}^{2}}+\frac{\mathbf{v}_{\perp}^{2}}{\mathrm{k} \boldsymbol{\theta}_{\perp}^{2}}\right]^{-(\mathrm{k}+1)}
$$

And associated parallel and perpendicular effective thermal speeds are

$$
\begin{gathered}
\boldsymbol{\theta}_{\|}=\left[\frac{2 \mathrm{k}-3}{\mathrm{k}}\right]^{1 / 2}\left(\frac{\mathrm{T}_{\|}}{\mathrm{m}_{\mathrm{s}}}\right)^{1 / 2} \\
\boldsymbol{\theta}_{\perp}=\left[\frac{2 \mathrm{k}-3}{\mathrm{k}}\right]^{1 / 2}\left(\frac{\mathrm{T}_{\perp}}{\mathrm{m}_{\mathrm{s}}}\right)^{1 / 2}
\end{gathered}
$$

Applying the approximation in electron-cyclotron range of frequencies. In this case, ion temperature are assumed $\mathrm{T}_{\mathrm{is}}=\mathrm{T}_{\mathrm{i} \|}=\mathrm{T}_{\mathrm{i}}$ and assumed to be magnetized with $\left|\omega_{\mathrm{r}}+\mathrm{i} \gamma\right|$ $<<\omega_{\text {cs }}$ while electrons are assumed to have $T_{\perp \mathrm{e}}>\mathrm{T}_{\| \mathrm{e}}$ and $\left|k_{\|} \alpha_{\|}\right|<<\left|\omega_{\mathrm{r}} \pm \omega_{\mathrm{cs}}+\mathrm{i} . \gamma\right|$. Equation (19) becomes: 
$\mathrm{D}\left(\mathrm{k}, \omega_{\mathrm{r}}+\mathrm{i} \gamma\right)=1-\frac{\mathrm{k}^{2} \mathrm{c}^{2}}{\omega_{\mathrm{r}}+\mathrm{i} \gamma}+\sum \frac{\mathrm{J}_{\mathrm{p}}\left(\lambda_{2}\right) \mathrm{J}_{\mathrm{q}}\left(\lambda_{3}\right)}{\alpha_{\perp \mathrm{s}}^{2}} \times$

$\left.\left[\left\{\frac{\omega_{\mathrm{i}}^{2}}{\omega_{\mathrm{ce}}^{2}}-\frac{\omega_{\mathrm{pi}}^{2}}{\left.\left(\omega_{\mathrm{r}}+\mathrm{i} \gamma \gamma\right) \pm \omega_{\mathrm{ce}}\right)}\right\} \mathrm{X}_{1} \mathrm{i} \frac{\omega_{\mathrm{pi}}^{2}}{\left(\omega_{\mathrm{r}}+\mathrm{i} \gamma \gamma\right)^{2}}\right\} \frac{\omega_{\mathrm{r}}+\mathrm{i} \gamma}{\mathrm{k}_{\|} \theta_{\| \mathrm{e}}}\left(\frac{\mathrm{k}-1}{\mathrm{k}}\right)^{1 / 2}\right] \times$

$\left[\left(\frac{\mathrm{k}-1}{\mathrm{k}-3 / 2}\right) \mathrm{Z}_{\mathrm{k}-1}\left(\left(\frac{\mathrm{k}-1}{\mathrm{k}}\right) 1 / 2 \xi_{\mathrm{e}}\right) \mathrm{A}_{\mathrm{T}}\right] \times$

$\left[\left\{1+\xi_{\mathrm{e}}\left(\left(\frac{\mathrm{k}-1}{\mathrm{k}}\right)^{1 / 2}\left(\frac{\mathrm{k}-1}{\mathrm{k}-3 / 2}\right) \mathrm{Z}_{\mathrm{k}-1}^{*}\left(\frac{\mathrm{k}-1}{\mathrm{k}}\right)^{1 / 2} \xi_{\mathrm{e}}\right)\right\}\right]-\frac{\omega}{\omega \pm \omega_{\mathrm{c}}} \frac{\omega_{\mathrm{pc}}^{2}}{\omega_{\mathrm{pw}}^{2}}$

Where $X_{1 \mathrm{i}}=\theta_{\perp \mathrm{i}}^{2}-\frac{v \Gamma_{\mathrm{xi}}}{\omega_{\mathrm{ci}}^{2}-v^{2}} \frac{\theta_{\perp \mathrm{i}}}{2} \sqrt{\pi}$

$\mathrm{X}_{1 \mathrm{e}}=\theta_{\perp \mathrm{e}}^{2}-\frac{v \Gamma_{\mathrm{xe}}}{\omega_{\mathrm{ce}}^{2}-v^{2}} \frac{\theta_{\perp \mathrm{e}}}{2} \sqrt{\pi}$

$\mathrm{A}_{\mathrm{T}}=\frac{\theta_{\perp \mathrm{e}}^{2}}{\theta_{\| \mathrm{e}}^{2}}-1$

Applying condition $\frac{\mathrm{k}^{2} \mathrm{c}^{2}}{\omega^{2}}>>1+\frac{\omega_{\mathrm{pi}}^{2}}{\omega_{\mathrm{ci}}^{2}}$ with $\mathrm{p}=1, \mathrm{n}=1$ and $\mathrm{q}=0$ we can get the growth rate and real freque

$$
\begin{gathered}
\mathrm{K}_{3}=1-\mathrm{X}_{3}+\mathrm{X}_{4}, \mathrm{~K}_{4}=\frac{\mathrm{X}_{3}}{1-\mathrm{X}_{3}+\mathrm{X}_{4}}, \widetilde{\mathbf{k}}=\frac{\mathbf{k}_{\|} \boldsymbol{\alpha}_{\|}}{\omega_{\mathrm{cs}}}, \\
\beta=\frac{\mathrm{K}_{\mathrm{B}} \mathrm{T}_{\|} \mu_{\mathrm{o}} \mathrm{n}_{\mathrm{o}}}{\mathrm{B}_{\mathrm{o}}^{2}} \quad \Gamma_{\mathrm{xs}}=\frac{\mathrm{eE}_{\mathrm{o}}}{\mathrm{m}_{\mathrm{s}}} \\
\mathrm{X}_{4}=\frac{-v}{\omega_{\mathrm{ce}}} \quad \delta=1+\frac{\mathrm{n}_{\mathrm{c}}}{\mathrm{n}_{\mathrm{w}}}\left(1+\mathrm{X}_{4}\right)
\end{gathered}
$$

\subsection{Case I}

When EMEC waves propagate parallel to magnetic field direction, the expression of growth rate and real frequency becomes:

$$
\begin{aligned}
& \frac{\gamma}{\omega_{\text {ce }}}=\frac{\frac{\sqrt{\pi}}{\widetilde{\mathbf{k}}}\left(\frac{(\mathrm{k}-1) ! \mathrm{k}^{\mathrm{k}-1 / 2}}{(\mathrm{k}-3 / 2) !}\right)\left(\mathrm{A}_{\mathrm{T}}-\mathrm{K}_{4}\right) \times}{1+\mathrm{X}_{4}+\frac{\mathrm{k}}{\mathrm{k}-3 / 2}\left[\frac{\left(1+\mathrm{X}_{4}\right) \widetilde{\mathbf{k}}^{2}}{2 \mathrm{~K}_{3}^{2}}+\frac{\widetilde{\mathbf{k}}^{2}}{\mathrm{~K}_{3}}\left(\mathrm{~A}_{\mathrm{T}}-\mathrm{K}_{4}\right)\right]-} \\
& \frac{\mathrm{X}_{1 \mathrm{e}}}{\mathrm{X}_{1 \mathrm{i}}} \mathrm{K}_{3}^{2}+\frac{(\delta-1) \mathrm{K}_{3}^{2}}{\left(1-\mathrm{X}_{3}\right)^{2}\left(1+\mathrm{X}_{4}\right)}
\end{aligned}
$$

$$
\mathrm{X}_{3}=\frac{\omega_{\mathrm{r}}}{\omega_{\mathrm{ce}}}=\frac{\widetilde{\mathbf{k}}^{2}}{\delta \beta}\left[\begin{array}{l}
\frac{\mathrm{X}_{1 \mathrm{e}}\left(1+\mathrm{X}_{4}\right)}{\mathrm{X}_{1 \mathrm{e}}-\mathrm{X}_{1 \mathrm{i}}\left(1+\mathrm{X}_{4}\right)}+ \\
\frac{\mathrm{A}_{\mathrm{T}} \beta \mathrm{X}_{1 \mathrm{e}}}{2\left(1+\mathrm{X}_{4}\right)\left(\mathrm{X}_{1 \mathrm{e}}-\mathrm{X}_{1 \mathrm{i}}\left(1+\mathrm{X}_{4}\right)\right)}
\end{array}\right]
$$

\subsection{Case II}

When EMEC waves propagate oblique to magnetic field direction, referring to equation no. (15), the reduced dielectric tensor in equation (18) becomes:

$$
\varepsilon_{11} \pm \varepsilon_{12}=\mathrm{N}^{2} \cos ^{2} \theta
$$

Using equation (23) and equation (19), following the similar approach, the expression of growth rate and real frequency becomes:

$$
\begin{aligned}
& \frac{\sqrt{\pi}}{\widetilde{\mathbf{k}} \cos \theta}\left(\frac{(\mathrm{k}-1) ! \mathrm{k}^{\mathrm{k}-1 / 2}}{(\mathrm{k}-3 / 2) !}\right) \times \\
& \frac{\gamma}{\omega_{\mathrm{ce}}}=\frac{\left(\mathrm{A}_{\mathrm{T}}-\mathrm{K}_{4}\right) \mathrm{K}_{3}^{3}\left\{-\left(\frac{\mathrm{k}^{3}}{\widetilde{\mathbf{k}} \cos \theta}\right)\right\}^{-2 \mathrm{k}}}{1+\mathrm{X}_{4}+\frac{\mathrm{k} \cos ^{2} \theta}{\mathrm{k}-3 / 2}\left[\frac{\left(1+\mathrm{X}_{4}\right) \widetilde{\mathbf{k}}^{2}}{2 \mathrm{~K}_{3}^{2}}+\frac{\widetilde{\mathbf{k}}^{2}}{\mathrm{~K}_{3}}\left(\mathrm{~A}_{\mathrm{T}}-\mathrm{K}_{4}\right)\right]-} \\
& \frac{\mathrm{X}_{1 \mathrm{e}}}{\mathrm{X}_{1 \mathrm{i}}} \mathrm{K}_{3}^{2}+\frac{(\delta-1) \mathrm{K}_{3}^{2}}{\left(1-\mathrm{X}_{3}\right)^{2}\left(1+\mathrm{X}_{4}\right)}
\end{aligned}
$$




$$
\mathrm{X}_{3}=\frac{\omega_{\mathrm{r}}}{\omega_{\mathrm{ce}}}=\frac{\widetilde{\mathbf{k}}^{2} \cos ^{2} \theta}{\delta \beta}\left[\begin{array}{l}
\frac{\mathrm{X}_{1 \mathrm{e}}\left(1+\mathrm{X}_{4}\right)}{\mathrm{X}_{1 \mathrm{e}}-\mathrm{X}_{1 \mathrm{i}}\left(1+\mathrm{X}_{4}\right)}+ \\
\frac{\mathrm{A}_{\mathrm{T}} \beta \mathrm{X}_{1 \mathrm{e}}}{2\left(1+\mathrm{X}_{4}\right)\left(\mathrm{X}_{1 \mathrm{e}}-\mathrm{X}_{1 \mathrm{i}}\left(1+\mathrm{X}_{4}\right)\right)}
\end{array}\right]
$$

\section{Results and Discussion}

\subsection{Plasma Parameter:}

Following plasma parameters are for the calculation of the growth rate and real frequency for the Lorentzian-Kappa driven EMEC waves in Uranian magnetosphere. Ambient magnetic field $\mathrm{B}_{0}=2.4 \times 10^{-10} \mathrm{~T}$, electric field $\mathrm{E}_{0}=4 \times 10^{-3} \mathrm{~V} / \mathrm{m}$ and electron density $\mathrm{n}_{\mathrm{o}}=5 \times 10^{4} \mathrm{~m}^{-3}, 6 \times 10^{4} \mathrm{~m}^{-3}$ and $4 \times 10^{4} \mathrm{~m}^{-3}$ has been considered. Temperature anisotropy $\mathrm{A}_{\mathrm{T}}$ is supposed to vary from 0.25 to 0.75 and $\mathrm{AC}$ frequency varies from $2 \mathrm{~Hz}$ to $10 \mathrm{~Hz}$ for background plasma. The thermal energy $\left(\mathrm{K}_{\mathrm{B}} \mathrm{T}_{11}\right)$ of background ions is taken to be $10 \mathrm{eV}$ and that of Maxwellian isotropic energetic beam electrons be $100 \mathrm{eV}$ [40]. As a result of the chosen parameters, we work in the frequency range of similar order, as discussed by [8].

\subsection{For Parallel Propagation}

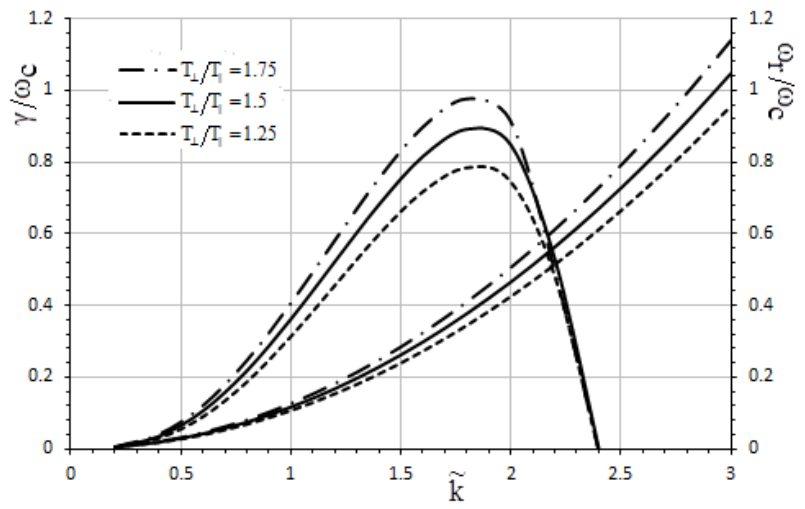

Fig.1: Variation of Growth Rate and Real Frequency with respect to $\tilde{k}$ for various values of $T_{\perp} / T_{\|}$at $n_{0}=4 \times 10^{4} \mathrm{~m}^{-3}, v$ $=2 \mathrm{~Hz}$ and other fixed plasma parameters.

Figure 1 shows the variation of growth rate and real frequency for different values of temperature anisotropy with respect to wave number. The derivation above explains that $\mathrm{T}_{\perp} / \mathrm{T}_{\|}=\mathrm{A}_{\mathrm{T}}$ +1 . So, for $T_{\perp} / T_{\|}=1.25$, maximum growth rate is $\gamma / \omega_{c}$ $=0.7858$. For $\mathrm{T}_{\perp} / \mathrm{T}_{\|}=1.5, \gamma / \omega_{\mathrm{c}}=0.8919$. Similarly, for $\mathrm{T}_{\perp} / \mathrm{T}_{\|}=1.75$, growth rate is $\gamma / \omega_{\mathrm{c}}=0.9771$. For various values of temperature anisotropy, the peak value appears at $\widetilde{\mathrm{k}}=1.8$. Therefore, as the value of ratio of temperature (perpendicular) to temperature (parallel) ie. $\mathrm{T}_{\perp} / \mathrm{T}_{\|}$increases growth rate as well as real frequency of EMEC waves increases when waves propagate parallel to ambient magnetic field. It can be said that as temperature anisotropy increases growth rate increases. The comparable work had been done for instability of parallel electromagnetic electron whistler-cyclotron modes using kappa distribution for terrestrial environments which differ significantly in terms of magnetic and electric field intensity of Uranian environment. [33]

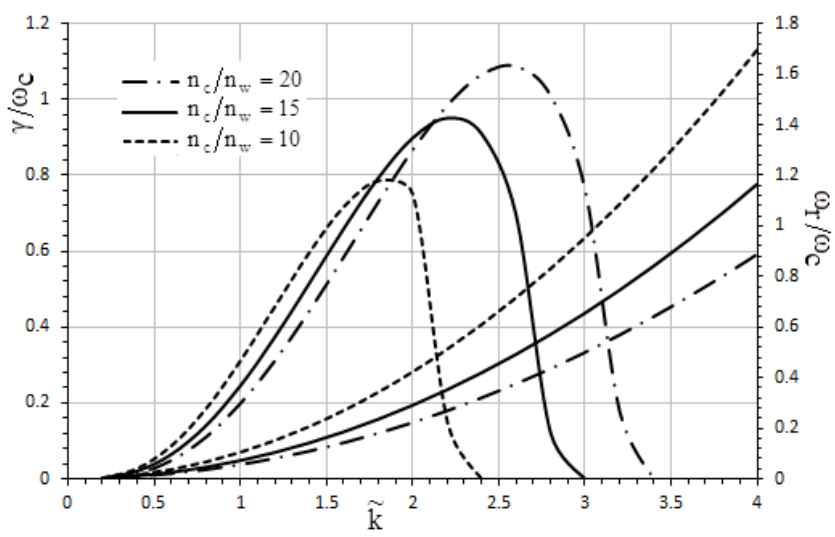

Fig. 2: Variation of Growth Rate and Real Frequency with respect to $\tilde{k}$ for various values of $n_{c} / n_{w}$ at $T_{\perp} / T_{\|}=1.25$, $\mathrm{n}_{\mathrm{o}}=4 \times 10^{4} \mathrm{~m}^{-3}$ and other fixed plasma parameters.

Figure 2 shows the variation of real frequency and growth rate with respect to $\widetilde{\mathbf{k}}$ for various values of ratio of cold electrons to warm electrons $\left(\mathrm{n}_{\mathrm{c}} / \mathrm{n}_{\mathrm{w}}\right)$. For $\mathrm{n}_{\mathrm{c}} / \mathrm{n}_{\mathrm{w}}=10$ and $\mathrm{n}_{\mathrm{c}} / \mathrm{n}_{\mathrm{w}}=15, \gamma / \omega_{\mathrm{c}}$ $=0.7858$ and 0.9508 at $\widetilde{\mathrm{k}}=1.8$ and 2.2 respectively. And at $\mathrm{n}_{\mathrm{c}} / \mathrm{n}_{\mathrm{w}}=20, \gamma / \omega_{\mathrm{c}}=1.0887, \widetilde{\mathrm{k}}=2.6$. The corresponding $\widetilde{\mathrm{k}}$ value maxima shifts from 1.8 to 2.6. Broadening of the wave spectrum and shift to higher wave number for higher ratio $\mathrm{n}_{\mathrm{c}} / \mathrm{n}_{\mathrm{w}}$ is clearly visible in the graph. Although real frequency of EMEC waves decreases distinctly under same condition. Thus even the minimal presence of such energetic electrons having a positive slope of distribution function $\partial f_{s o} / \partial v_{\perp}>0$ increases the growth of EMEC waves significantly. Similar results are drawn in case of whistler mode waves by a relativistic kappa-loss-cone distribution for magnetospheric space plasma [35]. 


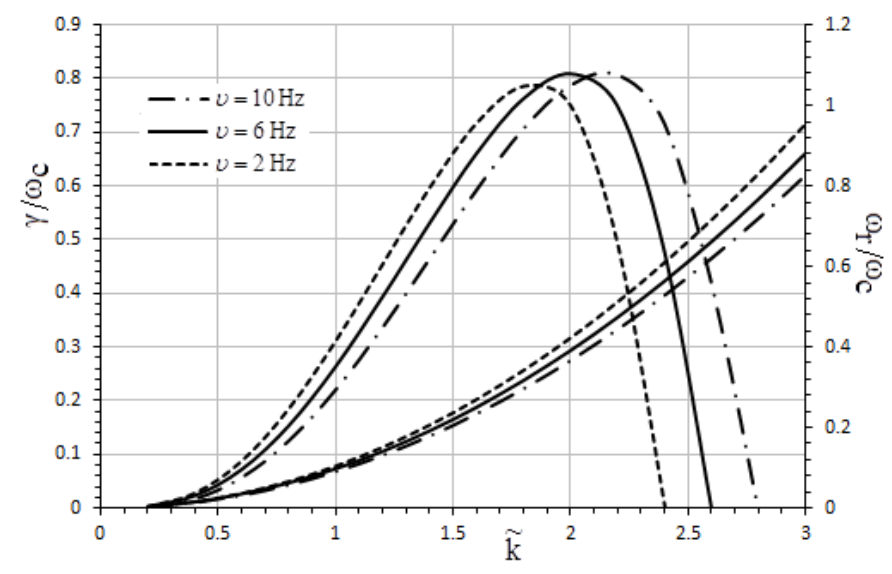

Fig. 3: Variation of Growth Rate and Real Frequency with respect to $\tilde{k}$ for various values of $A C$ frequency $\mathrm{U}$, at $T_{\perp} / T_{11}$ $=1.25, \mathrm{n}_{\mathrm{o}}=4 \times 10^{4} \mathrm{~m}^{-3}$ and other fixed plasma parameters.

Figure 3 shows the variation of growth rate and real frequency for different values of AC frequency $(U)$ with respect to wave number. For $\mathrm{U}=2 \mathrm{~Hz}$, maximum growth rate $\gamma / \omega_{\mathrm{c}}=0.7858$ at $\widetilde{\mathrm{k}}=1.8$ while propagating parallel to magnetic field direction. When $\mathrm{AC}$ frequency changes to $6 \mathrm{~Hz}$, peak occurs at $\widetilde{\mathrm{k}}=2.0$ with $\gamma / \omega_{\mathrm{c}}=0.8091$. And for $\mathrm{v}=10 \mathrm{~Hz}$, growth rate increases to $\gamma / \omega_{c}=0.8101$ at $\widetilde{k}=2.2$. The increase in growth rate is not supported by real frequency as can be seen in graph for higher AC field magnitude. In case of growth rate, broadening of wave spectrum at higher frequencies takes place along with shifting of peak value of $\widetilde{\mathrm{k}}$ from 1.8 to 2.2 due to Doppler shifting resonance condition occurring in magnetosphere of Uranus. The results are in accordance with normalized growth rate of whistler mode waves with perpendicular AC field considered in Uranian bow shock region [34].

\subsection{For Oblique Propagation}

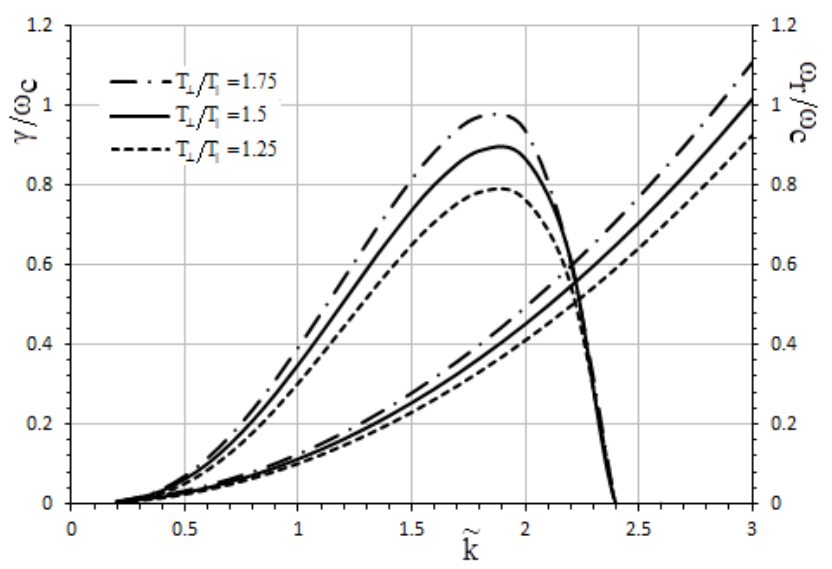

Fig. 4: Variation of Growth Rate and Real Frequency with respect to $\tilde{k}$ for various values of $T_{\perp} / T_{11}$ at $n_{0}=4 \times 10^{4} \mathrm{~m}^{-3}, v$ $=2 \mathrm{~Hz}, \theta=10^{\circ}$ and other fixed plasma parameters.

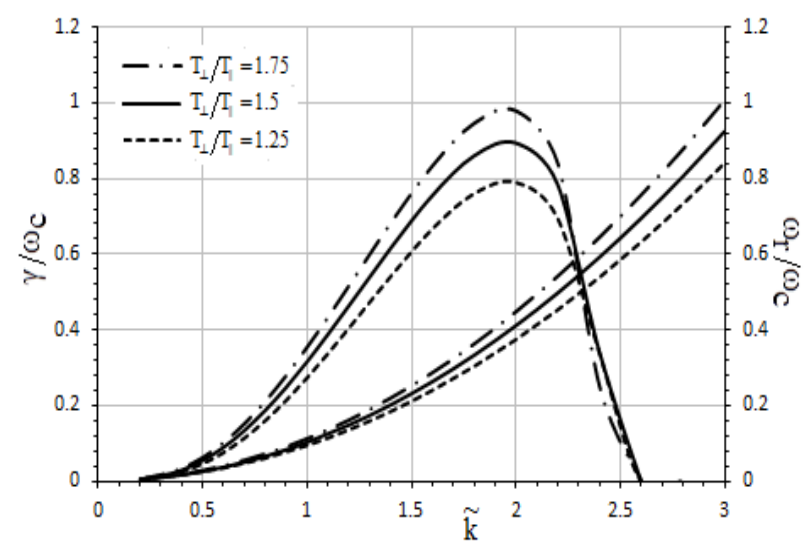

Fig. 5: Variation of Growth Rate and Real Frequency with respect to $\tilde{k}$ for various values of $T_{\perp} / T_{11}$ at $n_{0}=4 \times 10^{4} \mathrm{~m}^{-3}, v$ $=2 \mathrm{~Hz}, \theta=20^{\circ}$ and other fixed plasma parameters.

The perturbed particle trajectories in the presence of ac field having frequencies much smaller than plasma frequency and gyro frequencies have been evaluated and using the results of variation growth rate and real frequency, the graph is plot. Figure 4 shows the variation of growth rate and real frequency with respect to different temperature anisotropies when angle of propagation $(\theta)$ is $10^{\circ}$. From graph we can see that for value of ratio of temperature (perpendicular) to temperature (parallel) ie. $\mathrm{T}_{\perp} / \mathrm{T}_{\|}=1.25$, maximum growth rate $\gamma / \omega_{\mathrm{c}}=0.7812$ at $\widetilde{\mathrm{k}}$ $=1.8$. When $\mathrm{T}_{\perp} / \mathrm{T}_{\|}$becomes 1.5, peak is observed again at $\widetilde{\mathrm{k}}$ $=1.8$ with $\gamma / \omega_{c}=0.8866$. And for $T_{\perp} / T_{\|}=1.75$, growth is $\gamma / \omega_{c}=0.9721$ again at $\widetilde{k}=1.8$. So, growth rate increases with increase in the ratio of equivalent temperatures $T_{\perp} / T_{\|}$. Temperature anisotropy increases the growth rate by shifting the resonance conditions. Increase in temperature anisotropy also supports real frequency as it increases with increases in temperature anisotropy. The work done by [36] for oblique propagation of electromagnetic waves in kappa-Maxwellian plasma can also be referred to compare the effect of temperature anisotropy ratio on dispersion and damping of electromagnetic waves. Figure 5 analyses growth rate and real frequency for different values of $T_{\perp} / T_{\|}$at $\theta=20^{\circ}$. It can be seen that wave number is slightly shifter to higher number. For $\mathrm{T}_{\perp} / \mathrm{T}_{\|}=1.25$, 1.5 and 1.75 , the wave grows as $0.7877,0.8942$ and 0.9765 respectively. Although a slight decrease in magnitude of real frequency for increasing value of temperature anisotropy at $\theta$ $=20^{\circ}$ can be seen in figure. Uranus, being the example of 
oblique rotator with significant magnetic tilt and unusually large quadrupole moment [22, 41, 2], makes it interesting to study oblique propagation of waves in its magnetosphere.

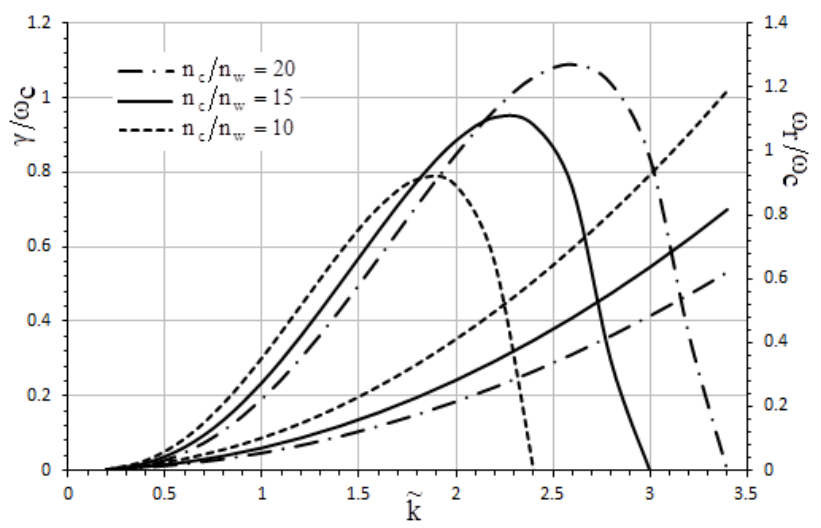

Fig. 6: Variation of Growth Rate and Real Frequency with respect to $\tilde{k}$ for various values of $n_{c} / n_{w}$ at $T_{\perp} / T_{11}=1.25, v$ $=2 \mathrm{~Hz}, \theta=10^{\circ}$ and other fixed plasma parameters.

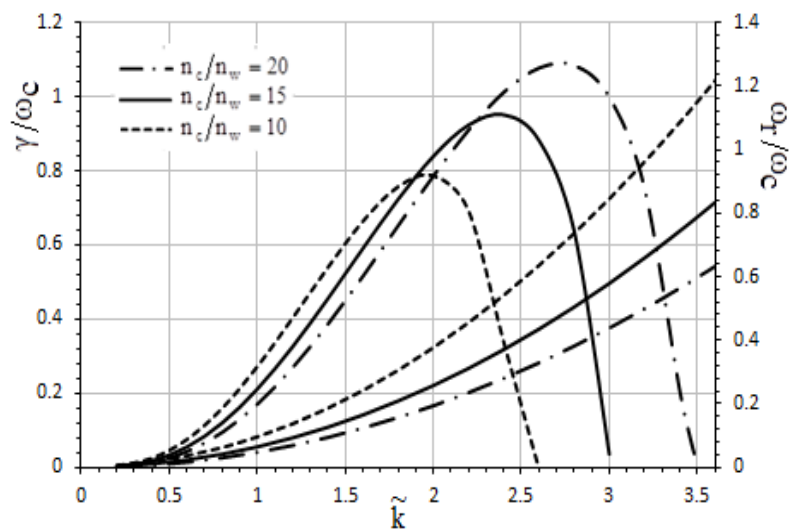

Fig. 7: Variation of Growth Rate and Real Frequency with respect to $\tilde{k}$ for various values of $n_{c} / n_{w}$ at $T_{\perp} / T_{11}=1.25, v$ $=2 \mathrm{~Hz}, \theta=20^{\circ}$ and other fixed plasma parameters.

Figure 6 shows the variation of real frequency and growth rate with respect to $\widetilde{\mathbf{k}}$ for various values of ratio of cold electrons to warm electrons $\left(\mathrm{n}_{\mathrm{c}} / \mathrm{n}_{\mathrm{w}}\right)$ for $\theta=10^{\circ}$. For $\mathrm{n}_{\mathrm{c}} / \mathrm{n}_{\mathrm{w}}=10$ and $\mathrm{n}_{\mathrm{c}} / \mathrm{n}_{\mathrm{w}}$ $=15, \quad \gamma / \omega_{\mathrm{c}}=0.7812$ and 0.9471 at $\widetilde{\mathrm{k}}=1.8$ and 2.2 respectively. And at $\mathrm{n}_{\mathrm{c}} / \mathrm{n}_{\mathrm{w}}=20, \gamma / \omega_{\mathrm{c}}=1.0907$ and $\widetilde{\mathrm{k}}=2.6$. The corresponding $\widetilde{\mathrm{k}}$ value maxima shifts from 1.8 to 2.6. When the propagation angle is increased to $20^{\circ}$ the growth rate and real frequency increases too. Figure 7 shows that at $\theta=20^{\circ}$ growth rate is 0.7877 for $n_{c} / n_{w}=10$, peak is seen at $\widetilde{k}=2.0$. In this case the most drastic shift in wave number is observed among all the graphs. For higher values of $n_{c} / n_{w}=15$ and 20 , maximum $\gamma / \omega_{\mathrm{c}}$ becomes is 0.9512 and 1.0779 at $\widetilde{\mathrm{k}}=2.4$ and 2.6 respectively. It can be concluded that increasing the power of perpendicular thermal velocity and positive slope of the distribution function has become appreciable competing sources of energies in the magnetosphere of Uranus. The result is in contrast with results given by [31] where oblique electromagnetic instabilities for a hot beam are discussed in magnetized plasma.

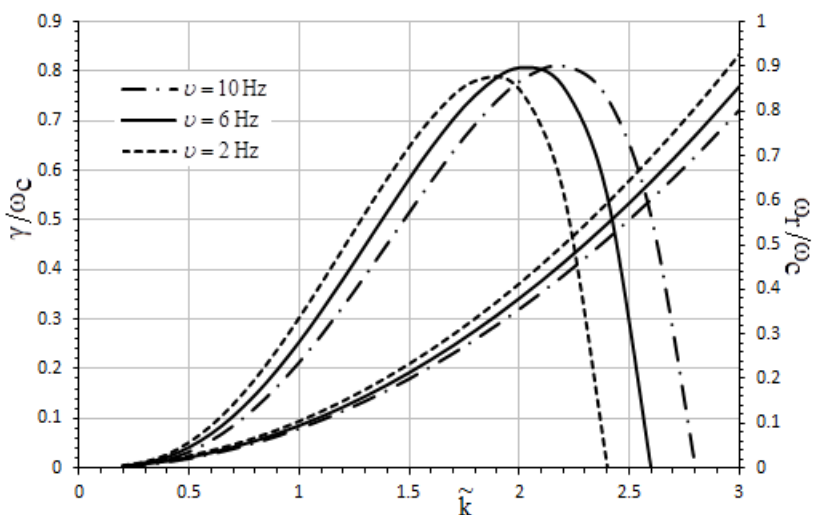

Fig. 8: Variation of Growth Rate and Real Frequency with respect to $\tilde{k}$ for various values of $A C$ frequency $\mathrm{U}$, at $T_{\perp} / T_{11}$ $=1.25, \mathrm{n}_{\mathrm{o}}=4 \times 10^{4} \mathrm{~m}^{-3}, \theta=10^{\circ}$ and other fixed plasma parameters

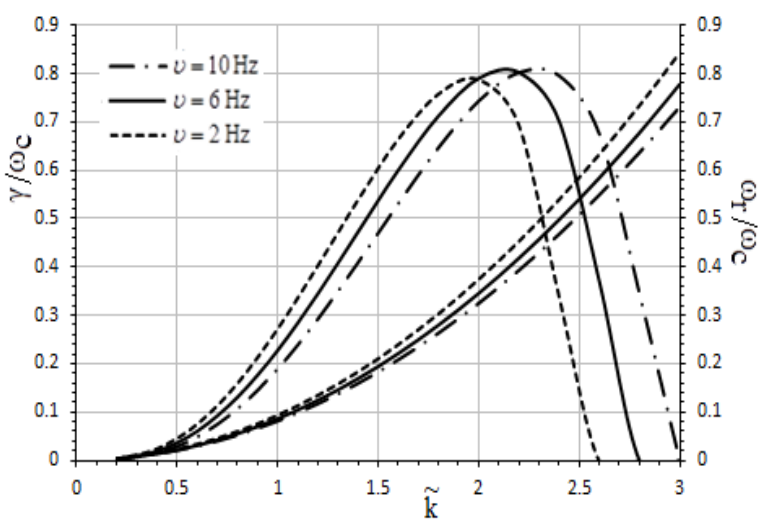

Fig. 9: Variation of Growth Rate and Real Frequency with respect to $\tilde{k}$ for various values of $A C$ frequency, $U$, at $T_{\perp} / T_{11}$ $=1.25, \mathrm{n}_{\mathrm{o}}=4 \times 10^{4} \mathrm{~m}^{-3}, \theta=20^{\circ}$ and other fixed plasma parameters

In figure $\mathbf{8}$ graph is plot between growth rate and real frequency versus wave number for different values of external AC field frequency at for $\theta=10^{\circ}$. As frequency changes from $2 \mathrm{~Hz}$ to 6 $\mathrm{Hz}$, maxima shifts from $\widetilde{\mathbf{k}}=1.8$ to 2.0 with change in growth rate from $\gamma / \omega_{\mathrm{c}}=0.7812$ to 0.8073 . When frequency becomes $10 \mathrm{~Hz}$, peak is seen at $\widetilde{\mathbf{k}}=2.2$ with $\gamma / \omega_{\mathrm{c}}=0.8103$. Therefore as external frequency increases growth rate increases but real frequency of EMEC waves decreases. Results can be comparable with work done by [28] where excitation of oblique whistler waves in magnetosphere of Earth and in interplanetary spaces at 1 A.U. is focused [28]. Also the results are analyzed 
at for $\theta=20^{\circ}$. The maximum growth rate seen in figure 9 is $0.7877,0.8023$ and 0.8033 at $U=2 \mathrm{~Hz}, 6 \mathrm{~Hz}$ and $10 \mathrm{~Hz}$ respectively. The difference in growth rate and increase in bandwidth is due to increased angle of propagation with respect to magnetic field.

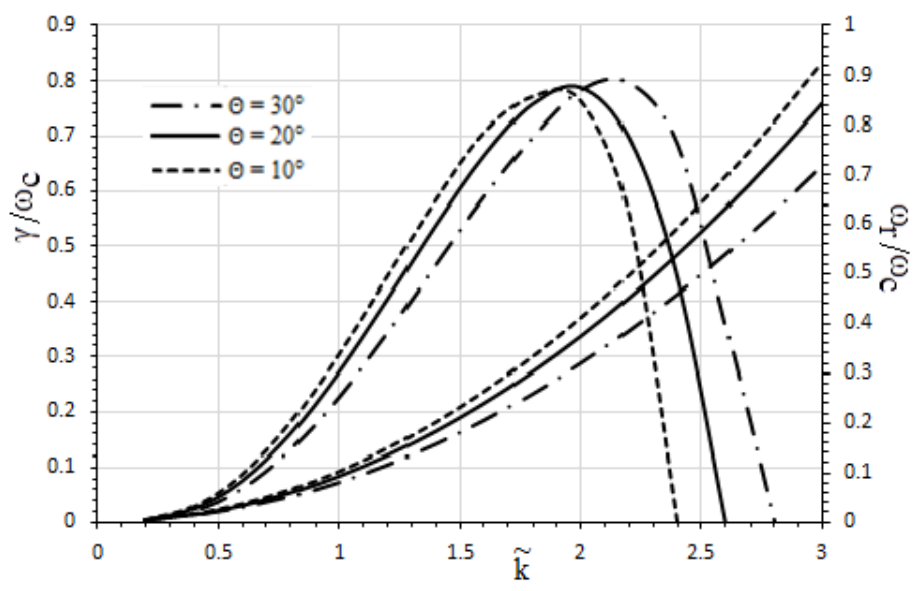

Fig. 10: Variation of Growth Rate and Real Frequency with respect to $\tilde{k}$ for various values of oblique angle, $\theta$, at $T_{\perp} / T_{11}$

$=1.25, \mathrm{n}_{\mathrm{o}}=4 \times 10^{4} \mathrm{~m}^{-3}, \mathrm{v}=2 \mathrm{~Hz}$ and other fixed plasma parameters.

The very large tilt of the magnetic dipole axis with respect to the planet's spin axis (sixty degree) combines to produce a dynamically curved magnetic field and magnetic field crossing. Figure 10 shows the effect of oblique angles on real frequency and growth rate of EMEC waves in the magnetosphere of Uranus. It is clearly seen in the graph that for $\theta=10^{\circ}$ the peak value $\gamma / \omega_{\mathrm{c}}=0.7812$ appears at $\widetilde{\mathrm{k}}=1.8$, for $\theta=20^{\circ}$ the peak value $\gamma / \omega_{\mathrm{c}}=0.7977$ appears at $\widetilde{\mathrm{k}}=2.0$ and for $\theta=30^{\circ}$ the peak value $\gamma / \omega_{\mathrm{c}}=0.8042$ appears again at $\widetilde{\mathrm{k}}=2.2$. And real frequency decreases with increase in value of oblique angle. As the value of oblique angle increases from $10^{\circ}$ to $30^{\circ}$ the growth rate increases. After $40^{\circ}$, electromagnetic component is replaced by electrostatic component as the non-resonant instability starts contributing [34].

\section{Conclusion}

In this paper electromagnetic electron cyclotron waves have been studied for Lorentzian Kappa distribution function in presence of external AC field in the magnetosphere of Uranus. The expression for dispersion relation, real frequency and growth rate is evaluated using method of characteristic solution, following the kinetic approach. Analyzing the effect of temperature anisotropy and number density of particles, it is inferred that these parameters are favorable for the growth of EMEC waves but in same conditions value of real frequency decreases for higher values of $\mathrm{AC}$ field, number density of beam and angle of propagation, in Uranian magnetosphere. As the value of temperature anisotropy and number density of particles increases, the growth rate increases. In case of oblique propagation, it is seen that bandwidth increases as the angle of propagation increases. Despite significant differences in magnetospheric parameters, the magnetosphere of Earth and Uranus both exhibit the presence EMEC waves with temperature anisotropy in presence of external AC field. Also, from the calculations conducted with Earth's a magnetospheric parameters, we conclude that EMEC waves in the magnetosphere of Earth, show their typical behavior for low values of wave number (of the order of $0<\widetilde{\mathbf{k}}<0.5$ ) than EMEC waves in the magnetosphere of Uranus (of the order of $0<\widetilde{\mathbf{k}}$ $<2.6$ ). The results above present a further insight into the nature of this instability condition for the ion-cyclotron waves by assuming ions as dominating energetic particles [42, 43] depending on geomagnetic activity in the magnetosphere of Uranus. In auroral acceleration region, the presence of hydrogen ion was reported by Voyager 2 spacecraft [44, 20]. Since the presence of different drifting ion species affect cold plasma dispersion relation and growth rate $[45,46]$, therefore the present basic model can be utilized to explain growth rate of electromagnetic ion-cyclotron waves by reconsidering the conditions of energetic charged particles [applied in equation no. 20] in the magnetosphere of Uranus or other space plasmas. These results are appropriate for applications to space plasma environments and various magnetospheric regimes for detailed comparative planetary study.

\section{Acknowledgement}

The authors are grateful to the Chairman, Indian Space Research Organisation (ISRO), Director and members of RESPOND program, ISRO, for the financial support. We are thankful to Dr. Ashok K. Chauhan (Founder President, Amity University) and Dr. Balvinder Shukla (Vice Chancellor, Amity University) for their immense encouragement. We also express our gratitude to the reviewers for their expert comments and fruitful suggestions for the manuscript.

\section{References}

[1] Behannon K.W., Acuna M.H., Burlaga L.F., Lepping R.P., Ness N.F., Neubauer F.M. 1977. Magnetic field experiment for Voyagers 1 and 2. Space Sci. Reviews 21(3): 235

[2] Ness N.F., Acuna M.H., Behannon K.W., Burlaga L.F., Connerney J.E.P., Lepping R.P. \& Neubauer F.M., 1986, Science, 233 no. 4759,85

[3] Gurnett D. A., Kurth W. S., Scarf F. L., Poynter R. L. 1986. First plasma wave observations at Uranus. Science 233: 106

[4] Scarf F.L., Gurnett D.A., Kurth W.S., Coroniti F.V., Kennel C.F., Poynter R.L. 1987. Plasma wave measurement in the magnetosphere of Uranus. $J$. Geophy. Res. 92 (A13): 15217 
[5] Kurth W.S., Gurnett D.A. 1991. Plasma waves in planetary magnetospheres. J. Geophys. Res. 96, 18977

[6] Kurth W.S., Barbosa D.D., Gurnett D.A., Scarf F.L. 1987. Electrostatic Waves in the Magnetosphere of Uranus. J. Geophy. Res. 92, A(13):15225

[7] Kurth W.S., Gurnett D.A., Scarf F.L. 1986. Sporadic Narrowband radio emissions from Uranus. $J$. Geophys. Res. 91: 11958

[8] Kurth W.S., Gurnett D.A., Scarf F.L., Mauk B.H. 1989. Plasma waves in magnetotail of Uranus. $J$. Geophy. Res. 94(A4): 3505

[9] Russell C.T., Song P., Lepping R.P. 1989. The Uranian magnetopause: Lessons from Earth. Geophys. Res. Letters 16(12): 1485

[10] Coroniti F.V., Kurth W.S., Scarf F.L., Krimigis S.M., Kennel C.F., Gurnett D.A. 1987. Whistler mode emissions in the Uranian radiation belts. J. Geophys. Res. 92: 15234

[11]Belmont G., Fontaine D., Canu P. 1983. Are equatorial electron cyclotron waves responsible for diffuse auroral electron precipitation? J. Geophys. Res. 88: 9163

[12]Roeder J.L. and Koons H.C. 1989. A survey of electron cyclotron waves in the magnetosphere and the diffuse auroral electron precipitation. J. Geophys. Res. 94: 2529

[13] Summers D., Tang R., Thorne R.M. 2009. Limit on stably trapped particle fluxes in planetary magnetospheres. J. Geophys. Res. 114: A10210

[14] Meredith N.P., Horne R.B., Thorne R.M., Anderson R.R. 2009. Survey of upper band chorus and ECH waves: Implications for the diffuse aurora. J. Geophys. Res. 114: A07218

[15] Stone E.C., Cooper J.F., Cummings A.C., McDonald F.B., Trainor J.H., Lal N., McGuire R., Chenette D.L. 1986. Energetic charged particles in the Uranian magnetosphere. Science 233(4759): 93

[16] Selesnick R.S. and Stone E.C. 1991, Energetic electrons at Uranus: Bimodal diffusion in a satellite limited radiation belt. J. Geophy. Res. 96 (A4): 5651

[17] Kennel C.F., Engelmann, F. 1966. Low-Frequency Whistler Mode. Phys. of Fluids 9: 2377.

[18] Shklyar D. and Matsumoto H. 2009. Oblique Whistler-Mode Waves in the Inhomogeneous Magnetospheric Plasma: Resonant Interactions with Energetic Charged Particles, Surv. Geophys.30: 55

[19]Cuperman S. 1981. Electromagnetic kinetic instabilities in multicomponent space plasmas: Theoretical predictions and computer simulation experiments. Revs. Geophys. Space Phys. 19: 307

[20] Krimigis S.M. et al. 1986. The magnetosphere Of Uranus: hot plasma and radiation environment. Science 233(4759): 97

[21] McNutt Jr. R.L., Selesnick R.S., Richardson J.D. 1987. Low-energy plasma observations in the magnetosphere of Uranus. J. Geophy. Res. 92(A5): 4399
[22] Kurth W. S. 1992. Comparative observations of plasma waves at the outer planets. Adv. Space Res. 12(8): 83

[23] Viñas A.F., Mace R.L., Benson R.F. 2005. Dispersion characteristics for plasma resonances of Maxwellian and Kappa distribution plasmas and their comparisons to the IMAGE/RPI observations. J. Geophy. Res. 110: A06202

[24]Pierrard V. and Lazar M. 2010. Kappa Distributions: Theory and applications in space plasma. Solar Physics 267, 153

[25] Hudson M.K., Clark J.T., Warren J.A. 1989. Inospheric dynamo theory for production of far ultraviolet emissions on Uranus. J. Geophys. Res. 94: 6517

[26] Pandey R.S., Kaur R., Kumar S., Kumar M. 2013. Study of VLF Mode Instability with AC Electric Field for Subtracted Bi-Maxwellian in the Magnetosphere of Uranus. J. Emerging Trends in Eng. And Applied Sci. 4(2): 201

[27] Pandey R.S., Kaur R. 2012. Generation of low frequency electromagnetic wave by injection of cold electron for relativistic and non-relativistic subtracted bi-maxwellian distribution with perpendicular AC electric field for magnetosphere of Uranus. Progress in Electromagnetics Res. B 45: 337

[28] Pandey R.S., Pandey R.P., Misra K.D. 2002. Temporal evolution of whistler mode instability due to cold plasma injection in the Presence of perpendicular AC electric field in the magnetosphere of Uranus. Earth, Moon and Planets 91: 209

[29] Tokar R.L. and Gary S.P. 1985. The whistler mode in a Vlasov plasma. Phys. of Fluids 28: 1063

[30] Gary S.P., 1985. Electrostatic instabilities in plasmas with two electron components. J. Geophys. Res. 90(A9): 8213

[31]Bret A., Dieckmann M.E., Deutsch C. 2006. Oblique electromagnetic instabilities for a hot relativistic beam interacting with a hot and magnetized plasma. Physics of Plasma 13: 082109

[32] Misra, K.D., Pandey, R.S. 1995. Generation of whistler emissions by injection of hot electrons in the presence of perpendicular AC electric field. $J$. Geophy. Res. 100(A10): 19405

[33]Lazar M., Poedts S., Schlickeiser R., 2011, Instability of the parallel electromagnetic modes in Kappa distributed plasmas - I. Electron whistler-cyclotron modes. Monthly notice of Royal Astronomical Society 410 (1): 663

[34]Pandey R.P., Singh K.M., Pandey R.S. 2001. A theoretical study of the whistler mode instability at the Uranian bow shock. Earth, Moon and Planets 87: 59

[35]Xiao F. 2006. Modelling energetic particles by a relativistic kappa loss cone distribution function in plasmas, Plasma Phys. Controlled Fusion 48:203 
[36] Cattaert T., Hellberg M.A., Mace R.L., 2007. Oblique propagation of electromagnetic waves in a kappaMaxwellian plasma. Physics of Plasma 14: 082111

[37] Pandey R.S., Misra K.D. 2002. Excitation of oblique whistler waves in magnetosphere and in interplanetary space at 1 A.U. Earth Planets Space 54: 159

[38] Ahirwar G. 2012. Study of electromagnetic electron cyclotron waves around plasma-pause region, Res. J. Engineering Sci. 1(6): 18

[39] Pandey R. S., Pandey R.P., Srivastava A.K., Dubey K., 2005, Indian Journal of Radio \& Space Physics, 34, 98

[40] Pandey R.S., Pandey R.P., Srivastava A.K., Karim S.M., Hariom, 2008. The electromagnetic ioncylclotron instability in the presence of AC electric field for lorentzian kappa. Progress in Electromagnetic Research M 1: 207

[41] Connerny J.E.P., Acuna M.H., Ness N.F., 1987, J. Geophys. Res., 92, A13, 15329

[42] Pandey R.S., Singh D.K. 2010. Study of electromagnetic ion cyclotron instability in magnetoplasma. Progress In Electromagnetics Research M 14: 147

[43] Tyagi R.K., Srivastava K.K., Pandey R.S. 2011. Analysis of electrostatic ion-cyclotron instability driven by parallel flow velocity shear. Surface Engineering and Applied Electrochemistry 47 (4): 370

[44] Cheng A.F., Krimigis S.M., Mauk B.H., Keath E.P., Maclennan C.G., Lanzerotti J.L., Paonessa M.T., Armstrong T.P. 1987. Energetic ion and electron phase space densities in the magnetosphere of Uranus. J. Geophys. Res. 92(A13): 15315
[45] Gomberoff L., Neira R. 1983. Convective growth rate of ion-cyclotron waves in a $\mathrm{H}^{+}-\mathrm{He}^{+}$, and $\mathrm{H}^{+}-\mathrm{He}^{+}-\mathrm{O}^{+}$ plasma. J. Geophys. Res. 88: 2170

[46] Gomberoff L., Gnavi G., Gratton F.T. 1996. Minor heavy ion electromagnetic beam-plasma interaction. $J$. Geophys. Res. 101: 13517 